ARTICLE

\title{
Host-inherent variability influences the transcriptional response of Staphylococcus aureus during in vivo infection
}

\author{
Robert Thänert', Oliver Goldmann¹, Andreas Beineke ${ }^{2} \&$ Eva Medina ${ }^{1}$
}

The rise of antibiotic resistance calls for alternative strategies to treat bacterial infections. One attractive strategy is to directly target bacterial virulence factors with anti-virulence drugs. The expression of virulence traits by pathogens is, however, not constitutive but rather induced by the level of stress encountered within the host. Here we use dual RNA sequencing (RNA-seq) to show that intrinsic variability in the level of host resistance greatly affects the pathogen's transcriptome in vivo. Through analysis of the transcriptional profiles of host and pathogen during Staphylococcus aureus infection of two mouse strains, shown to be susceptible $(A / J)$ or resistant $(\mathrm{C} 57 \mathrm{BL} / 6)$ to the pathogen, we demonstrate that the expression of virulence factors is dependent on the encountered host resistance. We furthermore provide evidence that this dependence strongly influences the efficacy of anti-virulence strategies, highlighting a potential limitation for the implementation of these strategies.

\footnotetext{
${ }^{1}$ Infection Immunology Research Group, Helmholtz Centre for Infection Research, Inhoffenstrasse 7, 38124 Braunschweig, Germany. ${ }^{2}$ Institute for Pathology, University of Veterinary Medicine Hannover, Bünteweg 17, 30559 Hannover, Germany. Correspondence and requests for materials should be addressed to E.M. (email: eva.medina@helmholtz-hzi.de).
} 
T he rise of antimicrobial resistance is one of the most challenging problems in modern medicine, causing an increase in morbidity and mortality associated with common bacterial infections ${ }^{1}$. While available antibiotics are loosing their effectiveness, the introduction of novel bactericidal or bacteriostatic antibiotics cannot be considered a long-term solution because it is eventually followed by the emergence of resistant bacterial clones that become increasingly prevalent under selective drug pressure. Consequently, there is a pressing need for new anti-infective agents that do not impose similar levels of selection pressure on pathogens as classical antibiotics. In this regard, alternative approaches based on attenuating bacterial pathogenesis by targeting bacterial virulence, the so-called 'anti-virulence' strategies, are emerging as promising tools for the treatment of infections ${ }^{2}$.

Bacterial pathogens express a large repertoire of different virulence factors to survive under the adverse conditions imposed by the host environment. Thus, anti-virulence strategies have been proposed that specifically target bacterial toxins produced by the pathogen to evade host defenses ${ }^{3}$, bacterial factors mediating adhesion to the host ${ }^{4}$, secretion systems ${ }^{5}$ as well as regulatory systems $^{6}$ and quorum-sensing signalling ${ }^{7}$. The key feature of anti-virulence drugs is the attenuation of the pathogen's virulence to aid clearance by the host's immune defenses ${ }^{2}$. These drugs seem attractive, because it is believed that not killing the pathogen directly exerts less selective pressure for the development of resistance ${ }^{2}$. However, such an approach will only confer therapeutic benefit if the targeted virulence factor(s) are actually expressed by the bacterium during infection and if the natural defense mechanisms of the host are strong enough to clear the pathogen, weakened by the anti-virulence treatment.

Bacterial pathogenesis, on the other hand, is strongly influenced by the strength of the host immune defense. For example, avirulent microorganisms can be pathogenic for immunocompromised hosts, whereas virulent microorganisms can be nonpathogenic in immune hosts ${ }^{8}$. This situation is further complicated by the fact that, in addition to the immune status, inherent characteristics of the host, such as the genetic background, significantly influence the capability of the immune system to overcome invading pathogens. Thus, the response to a specific pathogen can range from weak in susceptible hosts, causing severe infections, to strong in more resistant individuals, resulting in milder diseases. These differences imply that pathogens will encounter stronger immune pressure in resistant than in susceptible hosts and virulence factors that are essential for counteracting a weak immune response may not be the same as those required under stronger immune pressure in resistant hosts. Therefore, the dependence of virulence factor expression on host resistance is a potential limitation for the effectiveness of anti-virulence drugs.

Here we investigate how intrinsic variability of host resistance to a pathogen affects the expression of virulence factors needed to successfully infect the host. We use Staphylococcus aureus, a human pathogen that can cause severe invasive infections ${ }^{9}$ and is notorious for its capacity to develop antibiotic resistances. These characteristics make $S$. aureus one of the most dangerous and intractable infectious pathogens worldwide ${ }^{10}$. Despite numerous attempts to develop a vaccine that can prevent $S$. aureus infections, none of the vaccine candidates tested in clinical trials has succeeded so $\mathrm{far}^{11}$. This failure, in combination with the increase of antibiotic resistance, has lead to an intensification of efforts to search for alternative treatment approaches in recent years. In this regards, anti-virulence strategies targeting crucial pathogenicity factors produced by S. aureus during infection have been proposed as an attractive therapeutic option ${ }^{12,13}$. However, since the outcome of $S$. aureus infection is strongly influenced by the host factors such as racial origin, age and genetic makeup ${ }^{14,15}$, the search for anti-virulence targets in $S$. aureus has to consider the inherent variability of the host responses to infection. Similar to humans, variability in the host response to $S$. aureus has been also observed among different inbred strains of mice ${ }^{16}$. While some mouse strains (for example, $\mathrm{A} / \mathrm{J}$ and $\mathrm{DBA} / 2$ ) are very susceptible to $S$. aureus infection, C57BL/6 mice are highly resistant and survive a bacterial dose that rapidly kills mice from susceptible strains ${ }^{16}$. These differences in the capacity to control $S$. aureus infection provide a unique experimental system to explore the extent to which intrinsic host variability affects the expression of bacterial virulence factors during infection.

In this study, we use dual RNA sequencing (RNA-seq), and two mouse strains previously shown to display differential susceptibility to $S$. aureus infection (strain $\mathrm{A} / \mathrm{J}$ is susceptible and strain $\mathrm{C} 57 \mathrm{BL} / 6$ is resistant) $)^{16,17}$, to investigate how the intrinsic variability of host resistance to a pathogen affects the expression of virulence factors. Dual RNA-seq enables the simultaneous determination of the transcriptional response of the host and pathogen during infection and does not require physical separation of prokaryotic and eukaryotic RNA since the sequencing reads can be assigned to the host or to the pathogen genomes by in silico analysis ${ }^{18}$. Although dual RNA-seq has been successfully applied to characterize the transcriptional signature of bacteria and host in several in vitro infection systems ${ }^{14,15,19,20}$, the study presented here is one of the first using this technology in an in vivo system. Our results demonstrate the impact of the host genetic background on the transcriptional response of $S$. aureus during infection, and provide experimental evidence that host-dependent bacterial expression of virulence factors is a potential limitation for the efficacy of anti-virulence therapies.

\section{Results}

$\mathrm{C} 57 \mathrm{BL} / 6$ and $\mathrm{A} / \mathrm{J}$ mice differ in their resistance to $S$. aureus. For this study, we selected two strains of mice that differ in their susceptibility to $S$. aureus infection ${ }^{16,17}$. Whereas $\mathrm{A} / \mathrm{J}$ mice are very vulnerable to $S$. aureus, resulting in a significantly increased bacterial growth in the kidneys (Fig. 1a) and liver (Fig. 1b) and fatal infection outcome (Fig. 1c), C57BL/6 mice exhibit greater resistance to $S$. aureus and were capable to significantly restrict bacterial growth in the kidneys (Fig. 1a) and liver (Fig. 1b) with all mice surviving (Fig. 1c). Therefore, these two mouse strains provide an excellent tool for investigating the extent to which variability in the host response to infection affects the transcriptional response of $S$. aureus within the host.

Dual RNA-seq analysis of $S$. aureus and infected host tissue. A dual RNA-seq approach that enables simultaneous transcriptional profiling of bacteria and host tissue was used to characterize the host response to infection and investigate the impact of different levels of host resistance on the transcriptional response of $S$. aureus. The schematic representation of the experimental design is shown in Fig. 1d. Total RNA including host and pathogen RNA, was isolated from the kidneys of $\mathrm{A} / \mathrm{J}$ and $\mathrm{C} 57 \mathrm{BL} /$ 6 mice at $48 \mathrm{~h}$ of infection and analysed by Illumina deep sequencing. Between 9 and 21 millions reads were uniquely aligned to the reference genome of Mus musculus assembly GRCm38.p3 (GCA_000001635.5), while between 32,228 and 5 millions reads were uniquely mapped to the revised reference genome of S. aureus strain 8325-4 (ref. 21).

Transcriptome analysis of S. aureus-infected mice. Hierarchical clustering (Supplementary Fig. 1a) and principal component analysis (PCA) (Supplementary Fig. 1b) of gene expression datasets from infected $\mathrm{A} / \mathrm{J}$ and $\mathrm{C} 57 \mathrm{BL} / 6$ mice, as well as of 
a

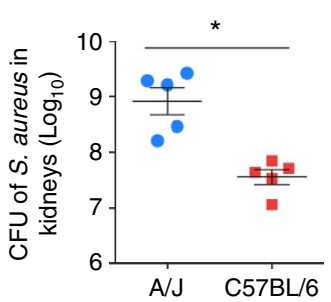

b

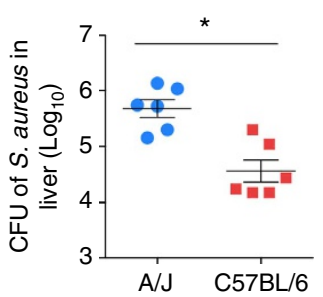

c

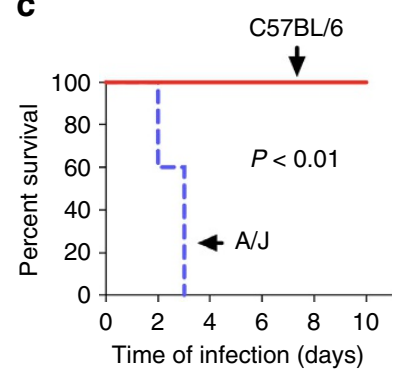

d

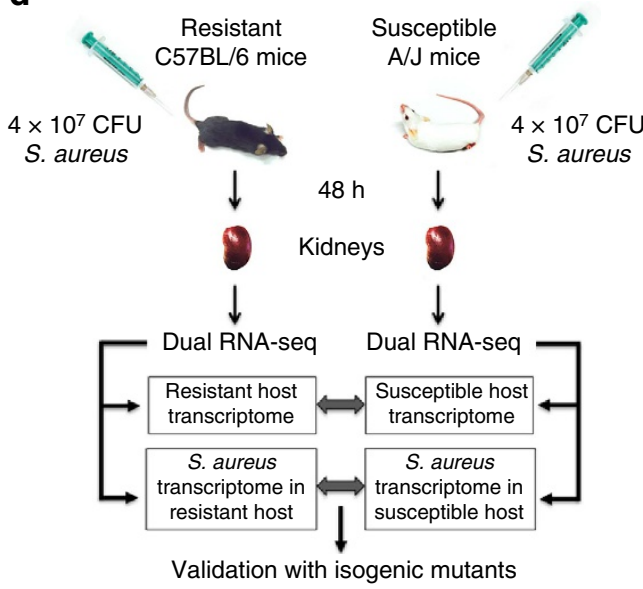

Figure 1 | A/J and C57BL/6 mice exhibit opposed levels of resistance to S. aureus. Bacterial loads in the kidneys (a) and liver (b) of $A / J$ and $C 57 B L / 6$ mice at $48 \mathrm{~h}$ after intravenous inoculation with $4 \times 10^{7} \mathrm{CFU}$ of $\mathrm{S}$. aureus SH1000. Each symbol represents the bacterial counts determined in an individual mouse and the horizontal lines represent the average $\pm \mathrm{s}$.d. for each mouse strain. One representative experiment out of three independent experiments is shown $\left(n=6\right.$, $t$-test, $\left.{ }^{\star} P<0.05\right)$. (c) Survival curves of $A / J$ and C57BL/6 mice intravenously infected with $4 \times 10^{7} \mathrm{CFU}$ of $\mathrm{S}$. aureus SH1000 ( $n=5$, log-rank test, $P<0.01$ ). (d) Schematic summary of the experimental design for dual RNA-seq analysis. Susceptible $\mathrm{A} / \mathrm{J}$ mice and resistant $\mathrm{C} 57 \mathrm{BL} / 6 \mathrm{mice}$ were infected intravenously with $4 \times 10^{7} \mathrm{CFU}$ of $\mathrm{S}$. aureus $\mathrm{SH} 1000$, their kidneys removed at $48 \mathrm{~h}$ after bacterial inoculation and subjected to dual RNA-seq analysis to simultaneously determine the gene expression profile of the host and pathogen in the same sample. The genes differentially expressed by S. aureus in $\mathrm{A} / \mathrm{J}$ and $\mathrm{C} 57 \mathrm{BL} / 6$ mice were identified and related to the infection-associated transcriptional response of the corresponding mouse strain. The effect of targeting a virulence factor differentially expressed by S. aureus $\mathrm{SH} 1000$ between infection of $\mathrm{A} / \mathrm{J}$ and $\mathrm{C} 57 \mathrm{BL} / 6$ mice was also determined.

tissue from uninfected control mice, demonstrated high withingroup reproducibility and substantial between-group differences. Pairwise comparison by permutational multivariate analysis of variance (PERMANOVA) revealed that the differences between all groups were statistically significant $(P<0.05$, Supplementary Table 1). Differential gene expression analysis using DESeq2 (ref. 22) identified a total of 5,540 differentially expressed genes (DEGs) between uninfected and $S$. aureus-infected A/J mice, of which 2,756 were found to be up-regulated in response to infection, while 2,784 were downregulated (Supplementary Data 1). In C57BL/6 mice, 3,559 genes were differentially expressed between uninfected and S. aureus-infected mice, of which 1,758 were upregulated and 1,801 were down-regulated in response to infection (Supplementary Data 2). The numbers of unique and overlapping DEGs between $S$. aureus-infected A/J and C57BL/6 mice are shown in Fig. 2a.

Functional classification of the DEGs using KEGG (Kyoto Encyclopedia of Genes and Genomes) pathway enrichment analysis, revealed that a large group of host genes with increased expression in response to infection in both $\mathrm{A} / \mathrm{J}$ and $\mathrm{C} 57 \mathrm{BL} / 6$ mice belonged to the groups 'cytokine-cytokine receptor interaction' and 'chemokine signalling pathway' (Fig. 2b). Particularly, genes encoding inflammatory cytokines such as IL- 6 , IL- $1 \alpha$, IL-1 $\beta$ and TNF- $\alpha$ as well as chemokines involved in the chemoattraction of monocytes/macrophages such as Cxcl1, Cxcl2 and $\mathrm{Cxcl} 3$, were upregulated in both $\mathrm{A} / \mathrm{J}$ and $\mathrm{C} 57 \mathrm{BL} / 6$ mice in response to S. aureus infection (Supplementary Data 1 and 2). Also, host genes encoding acute phase proteins such as Saa1, Saa2, haptoglobin and the calcium-binding proteins $\mathrm{S} 100 \mathrm{a} 8$ and S100a9 were highly induced in infected A/J and C57BL/6 mice. Although the global analysis of the transcriptional data suggested that a 'core' set of inflammation-related genes was highly expressed in both $\mathrm{A} / \mathrm{J}$ and $\mathrm{C} 57 \mathrm{BL} / 6$ mice in response to $S$. aureus infection, the average fold change of expression in this set of genes was markedly higher in infected $\mathrm{A} / \mathrm{J}$ than in infected C57BL/6 mice (Fig. 2). This suggested that $\mathrm{A} / \mathrm{J}$ mice developed a more intense systemic inflammation than C57BL/6 mice in response to $S$. aureus infection, which is indicative of severe sepsis leading to death. Besides the systemic hyperinflammation, the increased expression of the gene encoding the coagulation activator tissue factor (F3) and of the gene encoding the fibrinolysis inhibitor PAI-1 (Serpine1) in A/J mice (Supplementary Data 1), revealed a net pro-coagulant status that is typical for severe sepsis ${ }^{23}$. Altered coagulation, coupled with microvascular dysfunction occurring during sepsis, decreases tissue perfusion. This leads to perturbations of oxygen supply, resulting in tissue hypoxia and the activation of the hypoxiainducible factor alpha encoded by Hifla ${ }^{24,25}$. The significant induction of Hifla observed in $\mathrm{A} / \mathrm{J}$, but not in C57BL/6 mice, in response to infection (Supplementary Tables 2 and 3), indicated more severe tissue hypoxia in the kidneys of infected $\mathrm{A} / \mathrm{J}$ mice than in those of C57BL/6 mice. Furthermore, the genes encoding arginase $1(\mathrm{Arg} 1)$ and arginase $2(\mathrm{Arg} 2)$ were expressed to a greater extent in infected $\mathrm{A} / \mathrm{J}$ than in infected $\mathrm{C} 57 \mathrm{BL} / 6$ mice 
a
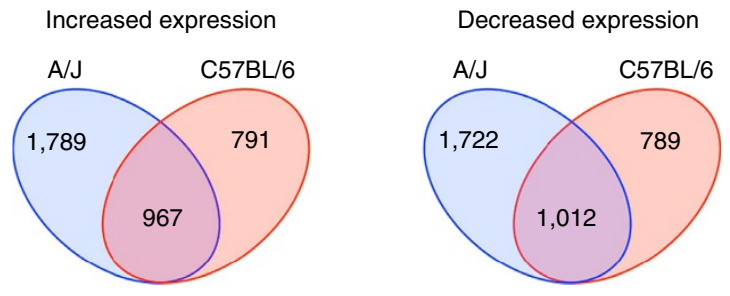

b

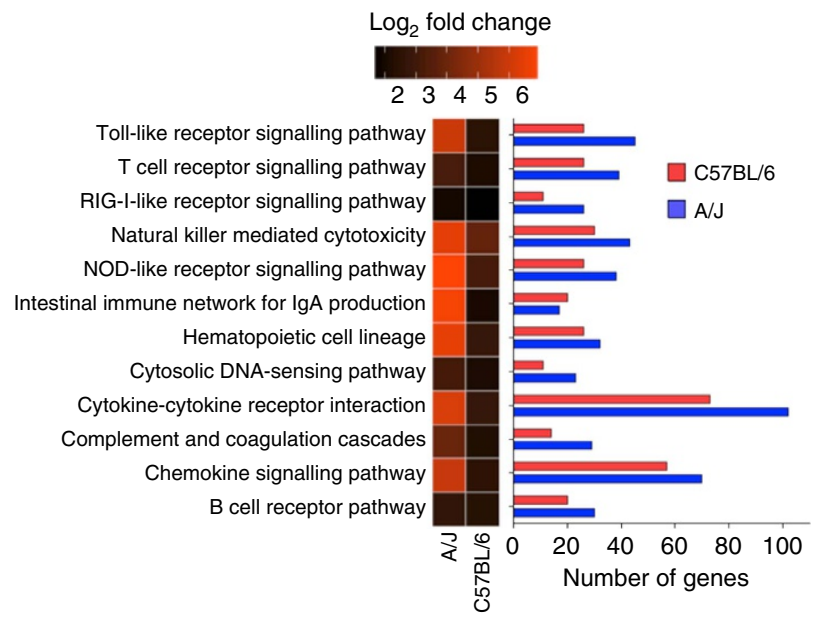

Figure 2 | Gene expression analysis of S. aureus-infected kidneys from $\mathbf{A} / \mathbf{J}$ and C57BL/6 mice. (a) Venn diagram showing the number of DEGs with increased (left) or decreased (right) expression in response to S. aureus infection that are unique or common between $\mathrm{A} / \mathrm{J}$ and $\mathrm{C} 57 \mathrm{BL} / 6$ mice. (b) Heat map of the mean $\log _{2}$-fold change of gene expression of the significantly up-regulated genes determined between $S$. aureus-infected versus uninfected $\mathrm{A} / \mathrm{J}$ and $\mathrm{C} 57 \mathrm{BL} / 6$ mice within the KEGG 'immune system' category (left part). The corresponding numbers of significantly up-regulated genes in response to $\mathrm{S}$. aureus infection in $\mathrm{A} / \mathrm{J}$ (blue bars) and $\mathrm{C} 57 \mathrm{BL} / 6$ (red bars) are shown in the right part of the figure.
(Supplementary Data 1). Arginase can contribute to endothelial cell dysfunction by depleting extra-cellular L-arginine and nitric oxide (NO) bioavailability, leading to endothelial nitric oxide synthase uncoupling and consequently to the production of high levels of harmful reactive oxygen species (ROS) ${ }^{26}$. Taken together, these findings suggest that the micro-environment in the infected tissue is highly different between $\mathrm{A} / \mathrm{J}$ and $\mathrm{C} 57 \mathrm{BL} / 6$ mice, which could significantly affect the expression of virulence determinants by $S$. aureus.

S. aureus transcriptome during infection of $\mathrm{A} / \mathrm{J}$ or $\mathrm{C} 57 \mathrm{BL} / 6$. In parallel, we analysed the transcriptome of $S$. aureus during the infection of resistant $\mathrm{C} 57 \mathrm{BL} / 6$ and susceptible $\mathrm{A} / \mathrm{J}$ mice to determine the impact of the different physiological conditions present at the site of infection on the pathogen's transcriptional response. Hierarchical ordination (Supplementary Fig. 2a) and PCA (Supplementary Fig. 2b) showed high within-group reproducibility, while PERMANOVA demonstrated that the transcriptional response of $S$. aureus infecting susceptible A/J mice differed significantly from that of $S$. aureus infecting resistant C57BL/6 mice (Supplementary Table 2). Transcript abundance was determined by normalizing the number of raw reads in each data set for gene length and expressed as transcripts per Kilobases per Million (TPM) (Supplementary Data 3). A total of 85 genes were identified as differentially expressed (probability value $\geq 0.95)$ by $S$. aureus between infection of $\mathrm{A} / \mathrm{J}$ and infection of C57BL/6 mice using NOISeq (Supplementary Data 4). Of those, transcripts of 20 genes were more abundant in $S$. aureus infecting A/J mice (Fig. 3a, Table 1, Supplementary Table 4), 65 genes exhibited greater expression in $S$. aureus during infection of C57BL/6 mice (Fig. 3a, Table 2, Supplementary Table 5) and 594 were expressed at a similar level by $S$. aureus in A/J and C57BL/6 mice (Fig. 3a, Supplementary Data 4).

One of the most prominent operons expressed by $S$. aureus to a greater extent during infection of $\mathrm{A} / \mathrm{J}$ mice, than during infection of $\mathrm{C} 57 \mathrm{BL} / 6$ mice, was the arc operon, which encodes the arginine

\section{Table 1 | DEGs between S. aureus infecting A/J and C57BL/6 mice with greater transcript abundance during infection of A/J} mice.

\begin{tabular}{|c|c|c|}
\hline Locus tag & Gene symbol & Description \\
\hline SAOUHSC_00845 & & Hypothetical \\
\hline SAOUHSC_02853 & & Hypothetical \\
\hline SAOUHSC_00371 & $y f l T$ & Hypothetical \\
\hline SAOUHSC_02964 & $\operatorname{arcR}$ & Hypothetical \\
\hline SAOUHSC_01477 & & Hypothetical \\
\hline SAOUHSC_01969 & $g v p P$ & Hypothetical \\
\hline SAOUHSC_00101 & $d r m$ & Phosphopentomutase \\
\hline SAOUHSC_01181 & $x y n A$ & Hypothetical \\
\hline SAOUHSC_02967 & $\operatorname{arcD}$ & Arginine/ornithine antiporter \\
\hline SAOUHSC_01191 & rpmB & $50 S$ ribosomal protein L28 \\
\hline SAOUHSC_00686 & & Hypothetical \\
\hline SAOUHSC_01803 & $\operatorname{aapA}$ & Hypothetical \\
\hline SAOUHSC_02862 & clpL & ATP-dependent Clp protease, ATP-binding subunit CIpC \\
\hline SAOUHSC_01403 & $\operatorname{cspA}$ & Cold shock protein \\
\hline SAOUHSC_02850 & $\operatorname{cid} B$ & Hypothetical \\
\hline SAOUHSC_01002 & $q 0 \times B$ & quinol oxidase AA3 subunit II \\
\hline SAOUHSC_01024 & graF & Hypothetical \\
\hline SAOUHSC_02702 & & Hypothetical \\
\hline SAOUHSC_02697 & tcyC & Amino acid $A B C$ transporter ATP-binding protein \\
\hline SAOUHSC_02665 & & Hypothetical \\
\hline
\end{tabular}


a

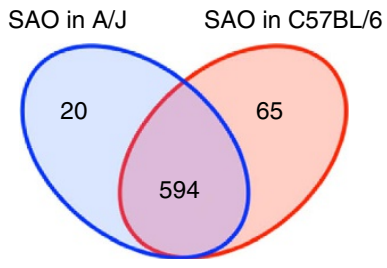

$\mathrm{SAO}$ in $\mathrm{A} / \mathrm{J}$ mice

$\mathrm{SAO}$ in C57BL/6 mice

b

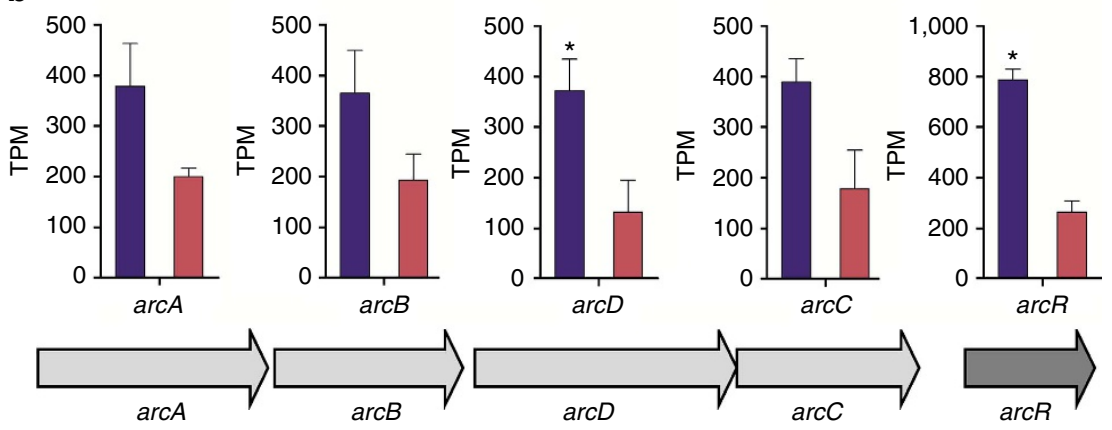

Figure 3 | Gene expression analysis of $\mathbf{S}$. aureus during infection of $\mathbf{A} / \mathbf{J}$ and $\mathbf{C 5 7 B L / 6}$ mice. (a) Venn diagram showing the number of unique and common expressed genes between S. aureus infecting $\mathrm{A} / \mathrm{J}$ and $\mathrm{C} 57 \mathrm{BL} / 6$ mice based on differential gene expression analysis determined with NOISeq. (b) Gene composition and organization of the genes of the ADI operon and their level of expression in S. aureus during infection of A/J (blue bars) or C57BL/6 (red bars) mice. Each bar represents the mean of TPM \pm s.d. of triplicates.

deiminase (ADI) system (Fig. 3b, Supplementary Data 4). The arc operon comprises the genes coding for arginine deiminase $(\operatorname{arc} A)$, ornithine transcarbamylase $(\operatorname{arcB})$, carbamate kinase $(\operatorname{arc} C)$, the arginine/ornithine antiporter $(\operatorname{arcD})$ and the transcriptional regulator ArcR $(\operatorname{arcR})$. These enzymes catalyse the conversion of arginine to ornithine, ammonia, and $\mathrm{CO}_{2}$, while producing ATP. This not only enables $S$. aureus to utilize arginine as an energy source under anaerobic conditions ${ }^{27}$ but also generates ammonia, which confers protection against acidic stress ${ }^{28}$. The expression of all genes of the ADI pathway was greater in $S$. aureus during infection of $\mathrm{A} / \mathrm{J}$ than during infection of $\mathrm{C} 57 \mathrm{BL} /$ 6 mice, even though only the differences in the level of $\operatorname{arcR}$ and $\operatorname{arcD}$ expression reached statistical significance (false discovery rate $(\mathrm{FDR})<0.05$, NOISeq analysis), (Fig. 3b, Supplementary Data 4).

Other genes, which were differentially expressed by $S$. aureus during infection of $\mathrm{A} / \mathrm{J}$ and $\mathrm{C} 57 \mathrm{BL} / 6$ mice, were those encoding proteins for amino acid transport and biosynthesis. Thus, transcripts of the genes encoding a hypothetical cysteine permease $(t c y C)$, a hypothetical lysine permease (lysP2), glutamate synthase $(g l t B, g l t D)$ and homoserine dehydrogenase $(d h o M)$ were more abundant in $S$. aureus during infection of $\mathrm{C} 57 \mathrm{BL} / 6$ mice than during infection of $\mathrm{A} / \mathrm{J}$ mice (Table 2 ). Because de novo amino acid synthesis pathways and amino acids transport systems are under control of catabolite repression in the presence of preferred carbon sources ${ }^{29}$ in $S$. aureus, the increased expression of the genes encoding these pathways during infection of C57BL/6 mice is consistent with more severe nutrient limitation in the tissue microenvironment of $\mathrm{C} 57 \mathrm{BL} / 6$ than in the tissue of $\mathrm{A} / \mathrm{J}$ mice. This nutrient limitation leads to de-repression of genes that enable $S$. aureus to use secondary carbon sources such as amino acids. Furthermore, genes encoding exoproteases such as staphopain (sspB2), serine proteases ( $s p A$, splA, splE and $s p l F)$, staphostatin B $(s s p)$, aureolysin (aur) and a cysteine protease $(\operatorname{ssp} B)$ were also expressed by $S$. aureus to a greater extent in C57BL/6 than in A/J mice (Fig. 4a). These proteases can generate peptides in the extracellular environment that can be imported by the bacterium via specialized transport systems and used to retrieve amino acids ${ }^{30}$. Besides their role in metabolism, extracellular proteases are potent virulence factors that help $S$. aureus to evade the host immune defenses ${ }^{31,32}$. Other genes encoding important virulence factors such as the immunodominant staphylococcal antigen B (isaB), the extracellular fibrinogen binding protein Efb (efb), which is involved in inhibition of phagocytosis ${ }^{33}$, the pore-forming cytolysin alpha-toxin ( $\left.h l a\right)$, and the amphipathic $\alpha$-helical phenol-soluble modulins psma1, psma2 and psma3, which can kill host cells by damaging the plasma membrane ${ }^{34}$, were also expressed by $S$. aureus to a higher extent during infection of $\mathrm{C} 57 \mathrm{BL} / 6$ mice (Fig. 4b). These findings indicate that $S$. aureus express greater levels of virulence factors during infection of C57BL/6 than during infection of A/J mice, which is most probably driven by the different growth phase of the bacteria in the two mouse strains.

The expression of these virulence factors is controlled by regulatory elements such as two-component regulatory systems (TCRS) and transcriptional regulatory systems in response to environmental cues encountered by the bacterium during infection ${ }^{35}$. The staphylococcal quorum-sensing system accessory gene regulator (agr) was found highly expressed by S. aureus during infection of both A/J and C57BL/6 mice. The $a g r$ system comprises two divergent transcripts, RNAII and RNAIII, which are under the control of two distinct promoters, P2 and P3, respectively ${ }^{36}$. RNA II encodes the quorum-sensing elements AgrB, AgrD, AgrC and AgrA that represent an autocatalytic sensory transduction system. RNAIII encodes delta-hemolysin $(h l d)^{37}$ and is a major regulator of virulence factors in S. aureus, inducing the transcription of various extracellular proteases and toxins ${ }^{38}$. Although the genes encoding the agr P2 operon 
Table 2 | DEGs between S. aureus infecting A/J and C57BL/6 mice with greater transcript abundance during infection of C57BL/6 mice

\section{Locus tag}

SAOUHSC_02260

SAOUHSC_00411.1

SAOUHSC_02566

SAOUHSC_02971

SAOUHSC_00435

SAOUHSC_01788

SAOUHSC_00987

SAOUHSC_00248

SAOUHSC_02571

SAOUHSC_00427

SAOUHSC 02941

SAOUHSC_01001

SAOUHSC_00964

SAOUHSC_00401

SAOUHSC 00717

SAOUHSC_00741

SAOUHSC_01942

SAOUHSC_00083

SAOUHSC_00348

SAOUHSC_00436

SAOUHSC 00051

SAOUHSC_00411.2

SAOUHSC_01121

SAOUHSC 00272

SAOUHSC 00801

SAOUHSC_01935

SAOUHSC_02369

SAOUHSC 00268

SAOUHSC_01110

SAOUHSC_01688

SAOUHSC 02855

SAOUHSC_02762

SAOUHSC_02114

SAOUHSC 02372

SAOUHSC_02430

SAOUHSC_01320

SAOUHSC_00986

SAOUHSC_00411.3

SAOUHSC_02112

SAOUHSC_02972

SAOUHSC_02885

SAOUHSC_01326

SAOUHSC_02127

SAOUHSC 01936

SAOUHSC_00728

SAOUHSC_00625

SAOUHSC 02763

SAOUHSC_00988

SAOUHSC_00711

SAOUHSC_00561

SAOUHSC 02550

SAOUHSC_00875

SAOUHSC_01359

SAOUHSC 01192

SAOUHSC_02887

SAOUHSC_02254

SAOUHSC 02485

SAOUHSC_01462

SAOUHSC_00367

SAOUHSC 01062

SAOUHSC_00893

SAOUHSC_00144

SAOUHSC_00652

SAOUHSC 02883

SAOUHSC_01431
Gene symbol

hld

psma1

sarR

aur

gltB

thrs

$\operatorname{ssp} B$

lytM

ssaA

sle1

nrdG

goxA

saeP

nrdl

splA

sbnl

rpsF

gltD

psma2

hla

$\sec G$

spIF

rpoE

efb

lepA

amiD2

dagK

hts A

dhoM

sspC

psma3

is $a B$

lysP2

sspB2

splE

mnhA

opp-1F

sspA

vraX

FdhD

ndh2

mprF

$v f r A$

isa $A$

groEL
rpoA

gps $B$

tcy $P$

namA

ausA

fhuA

ssaA

$m s r B$
Description

Delta-hemolysin

Alpha phenol soluble modulin

Hypothetical

Zinc metalloproteinase aureolysin

Glutamate synthase large subunit

Threonyl-tRNA synthetase

Cysteine protease

Peptidoglycan hydrolase

Secretory antigen

Autolysin

Hypothetical

Quinol oxidase subunit

Hypothetical

Hypothetical

Hypothetica

Ribonucleotide reductase stimulatory protein

Serine protease SplA

Hypothetical

30 S ribosomal protein S6

Glutamate synthase subunit beta

1-phosphatidylinositol phosphodiesterase

Alpha phenol soluble modulin

Alpha-hemolysin

Hypothetical

Preprotein translocase subunit SecG

Serine protease SpIF

DNA-directed RNA polymerase subunit delta

Hypothetical

Fibrinogen-binding protein-like protein

GTP-binding protein LepA

LysM domain-containing protein

Hypothetica

Putative lipid kinase

Hypothetical

$A B C$ transporter periplasmic binding protein

Homoserine dehydrogenase

Cysteine protease

Alpha phenol soluble modulin

Hypothetical

Immunodominant antigen B

Hypothetical

Hypothetical

Staphopain thiol proteinase

Serine protease SplE

Hypothetical

Putative monovalent cation/ $\mathrm{H}+$ antiporter subunit $\mathrm{A}$

Peptide ABC transporter ATP-binding protein

Glutamyl endopeptidase

Hypothetical

Hypothetical

Formate dehydrogenase accessory protein

Hypothetica

Hypothetical

Hypothetical

Immunodominant antigen $\mathrm{A}$

chaperonin GroEL

DNA-directed RNA polymerase subunit alpha

Hypothetical

Hypothetical

Hypothetical

FMN oxidoreductase

Hypothetical

Iron compound $\mathrm{ABC}$ transporter ATP-binding protein

LysM domain-containing protein

Methionine sulfoxide reductase $B$

DEG, differentially expressed gene.

The complete data of the differentially expressed genes with higher expression by S. aureus during infection of the resistant C57BL/6 mice are displayed in Supplementary Table 5.

(agrBDCA) were expressed by $S$. aureus to a similar level in both mouse strains, the expression level of RNAIII/hld was greater during infection of C57BL/6 mice (Fig. 4c, Supplementary Data 4). The gene encoding the transcriptional regulator SarR was also upregulated by $S$. aureus infecting C57BL/6 mice (Fig. 4c, Supplementary Data 4). This could explain the higher level of transcripts encoding proteases and toxins detected in
S. aureus during infection of C57BL/6 mice, since both RNAIII (ref. 38) and SarR ${ }^{39}$ activate their transcription.

Antimicrobial peptides (AMPs) are an important part of the host innate immune defense against $S$. aureus by directly impairing the integrity of the bacterial cell wall ${ }^{40}$. The bacterial gene encoding phosphatidylglycerol lysyltransferase (mprF), which is part of the cell wall stress stimulon and mediates 
resistance to cationic AMPs, by reducing the negative charge of the membrane surface ${ }^{41}$, was expressed by $S$. aureus to a larger extent during infection of resistant $\mathrm{C} 57 \mathrm{BL} / 6$ than during infection of A/J mice. Similarly, other genes of the cell wall stress stimulon including $v r a X$, autolysin encoding genes (ssaA, lytM, amiD2 and isaA), genes involved in cell separation ( $g p s B$ and $s l e 1$ ), groEL encoding a chaperonin, and the lipoteichoic acid synthase encoding gene ltaS exhibited greater expression levels in $S$. aureus during infection of $\mathrm{C} 57 \mathrm{BL} / 6$ mice than during infection of $\mathrm{A} / \mathrm{J}$ mice. The higher induction of these genes might reflect an adaptive response of $S$. aureus to keep cell wall integrity when confronted with high levels of cell wall damaging agents in the tissue of C57BL/6 mice.

Notably, genes related to central metabolism, iron acquisition, general and oxidative stress responses were expressed by $S$. aureus to a similar extent during infection of $\mathrm{A} / \mathrm{J}$ and $\mathrm{C} 57 \mathrm{BL} / 6$ mice (Supplementary Data 4), indicating that their expression was not influenced by the levels of host resistance to infection.

The RNA-seq results were validated in a selected set of genes from host and pathogen by quantitative reverse transcriptionPCR (RT-PCR; Supplementary Fig. 3) and at the protein level by ELISA (Supplementary Fig. 4).

Effect of decreased host resistance on $S$. aureus transcription. Next, we determined if depressing specific mechanisms of host resistance in $\mathrm{C} 57 \mathrm{BL} / 6$ mice affected the transcriptional response of $S$. aureus during infection. First, we hypothesized that apolipoprotein $B(A p o B)$, the major structural protein of very lowdensity (VLDL) and low-density lipoproteins (LDL), contributes to the resistance of C57BL/6 mice to $S$. aureus in our infection model, because the gene encoding Apo B (Apob) was expressed to a greater extent in the kidneys of C57BL/6 than in the kidneys of A/J mice at both gene expression (Fig. 5a) and protein level (Fig. 5b), before and after infection (Fig. 5c). Furthermore, ApoB has been shown to contribute to the host defense against $S$. aureus in experimental models of $\operatorname{skin}^{42}$ and respiratory ${ }^{43}$ infection by antagonizing the agr quorum sensing system of $S$. aureus. To validate this hypothesis, A/J and C57BL/6 mice were treated with 4-aminopyrazolopyrimidine (4-APP), a drug that impairs low-density lipoprotein secretion ${ }^{44}$, and subsequently infected intravenously with $S$. aureus. Whereas inhibition of ApoB rendered C57BL/6 mice more susceptible to $S$. aureus, demonstrated by the significantly higher bacterial loads in kidneys at $48 \mathrm{~h}$ infection ( $P$-value $<0.05, t$-test), (Fig. $5 \mathrm{~d}$ ), it did not affect the level of susceptibility of A/J mice (Fig. $5 \mathrm{~d}$ ). Next, we investigated if the reduced resistance in $\mathrm{C} 57 \mathrm{BL} / 6$ mice after attenuation of ApoB secretion impacted the transcriptional response of $S$. aureus during infection. For this purpose, we compared the expression of a set of virulence-related genes in $S$. aureus infecting 4-APP-treated $\mathrm{C} 57 \mathrm{BL} / 6$ mice with the expression of the same set of genes in $S$. aureus infecting C57BL/6 mice treated with vehicle alone. The results show that the level of expression of all genes tested hld/RNAIII, sarR, hla, sspA, aur, vraX and gltB was decreased by 4-APP-treatment, while the expression of the hypothetical alanine permease (aapA) was higher in $S$. aureus infecting 4-APP-treated than in vehicletreated C57BL/6 mice (Table 3). These genes were also found to be differentially expressed between $S$. aureus infecting C57BL/6 mice and $S$. aureus infecting C57BL/6 mice deficient in the expression of MyD88, an adaptor molecule that is essential for the signalling of IL-1R/TLR family (Table 3). Since MyD88-deficient mice are more susceptible to $S$. aureus than wild type C57BL/6 mice ${ }^{45}$, these observations further demonstrated the remarkable influence of the level of host resistance on the transcriptional response of $S$. aureus during infection.
Target expression affects efficacy of anti-virulence approaches. After having demonstrated the influence of the levels of host resistance on both the quality and quantity of $S$. aureus transcriptional response, we sought to determine the consequences of this dependence on the effectiveness of anti-virulence strategies. For this purpose, we assessed the effect of neutralizing a virulence factor that differed in expression between $S$. aureus infecting $\mathrm{A} / \mathrm{J}$ and C57BL/6 mice on the bacteria fitness during infection. We chose aureolysin, which has been shown to be important for full virulence of $S$. aureus in experimental infection models ${ }^{46,47}$ and was expressed to a significantly greater extent by $S$. aureus during infection of $\mathrm{C} 57 \mathrm{BL} / 6$ than during infection of $\mathrm{A} / \mathrm{J}$ mice in our study (FDR $<0.05$, NOISeq analysis). $\mathrm{C} 57 \mathrm{BL} / 6$ and $\mathrm{A} / \mathrm{J}$ mice were simultaneously challenged with wild type and an aureolysindeficient strain of $S$. aureus (wild type, $\Delta a u r$ ) and the amount of each bacterial strain was determined in the kidneys of infected mice at $48 \mathrm{~h}$ of infection. The overall amount of $S$. aureus bacteria counting both wild type and $\Delta a u r$ strains was significantly greater $(P=0.0138, t$-test $)$ in the kidneys of $\mathrm{A} / \mathrm{J}\left(1.3 \times 10^{8} \pm 4.3 \times 10^{7}\right)$ than in the kidneys of C57BL/6 mice $\left(1.03 \times 10^{7} \pm 5.4 \times 10^{6}\right)$. Moreover, while a lower amount of $\Delta$ aur than wild type $S$. aureus was recovered from $\mathrm{C} 57 \mathrm{BL} / 6$ mice, the amount of $\Delta a$ aur recovered from A/J mice was comparable to that of wild type strain (Fig. 6a). Thus, the $\Delta a u r$ had a competitive disadvantage when coadministered with wild type $S$. aureus in C57BL/6 mice (mean competitive index for $\Delta$ aur/wild type $S$. aureus of 0.013 ), while $\Delta$ aur and wild type $S$. aureus were equally competitive after co-administration in $\mathrm{A} / \mathrm{J}$ mice (mean competitive index for $\Delta$ aur/wild type $S$. aureus of 1.595) (Fig. 6b). Taken together, these results indicate that the efficacy of targeting a virulence factor by anti-virulence strategies will strongly depend on its level of expression by the pathogen during infection, which in turn is highly influenced by the intrinsic levels of host resistance to infection.

\section{Discussion}

Anti-virulence strategies based on attenuation of bacterial pathogenesis by the specific inhibition of virulence factors essential for the pathogen's survival during infection ${ }^{48}$, have received increasing attention as novel treatment options for infections caused by antibiotic-resistant pathogens ${ }^{48}$. The concept of anti-virulence therapy is still very much in its infancy and therefore more research is needed to explore its practicability. One important aspect that should be considered carefully when designing anti-virulence strategies is that the expression of virulence traits by the pathogens is not constitutive but rather influenced by the specific environment encountered during infection. Consequently, absent expression of the targeted virulence factors could render anti-virulence strategies completely ineffective. Therefore, it is essential to understand the impact of the wide-ranging, inter-individual variation of the host response on the pathogen's expression of virulence determinants during infection.

Our study supports the idea that the host genetic background affects the transcriptional response of $S$. aureus during infection. The limited capability of the immune defense mechanisms of susceptible A/J mice to control $S$. aureus growth led to the development of an intense inflammatory response, apparent by the disproportionate expression of inflammatory cytokines and damage-associated molecular patterns. Transcriptional reprogramming of the eukaryotic cells, possibly resulting from the concomitant accumulation of acidic products and the lowered oxygen tension (hypoxia) in the infected tissue, involved the induction of Hifla that encodes the central mediator of transcriptional responses to hypoxia HIF- $1 \alpha^{49,50}$. Therefore, the 
a

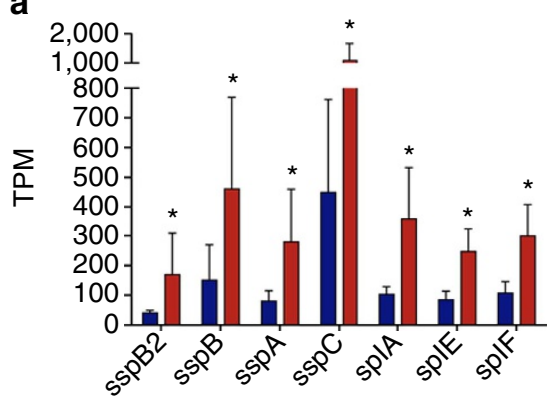

b

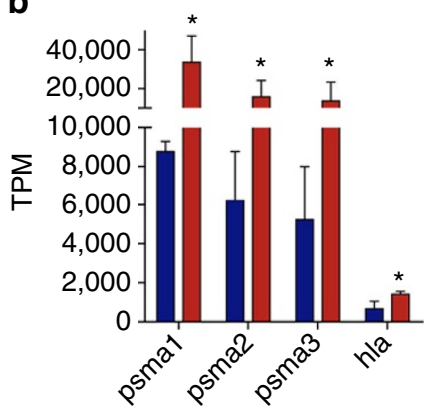

SAO in A/J mice

SAO in C57BL/6 mice

C
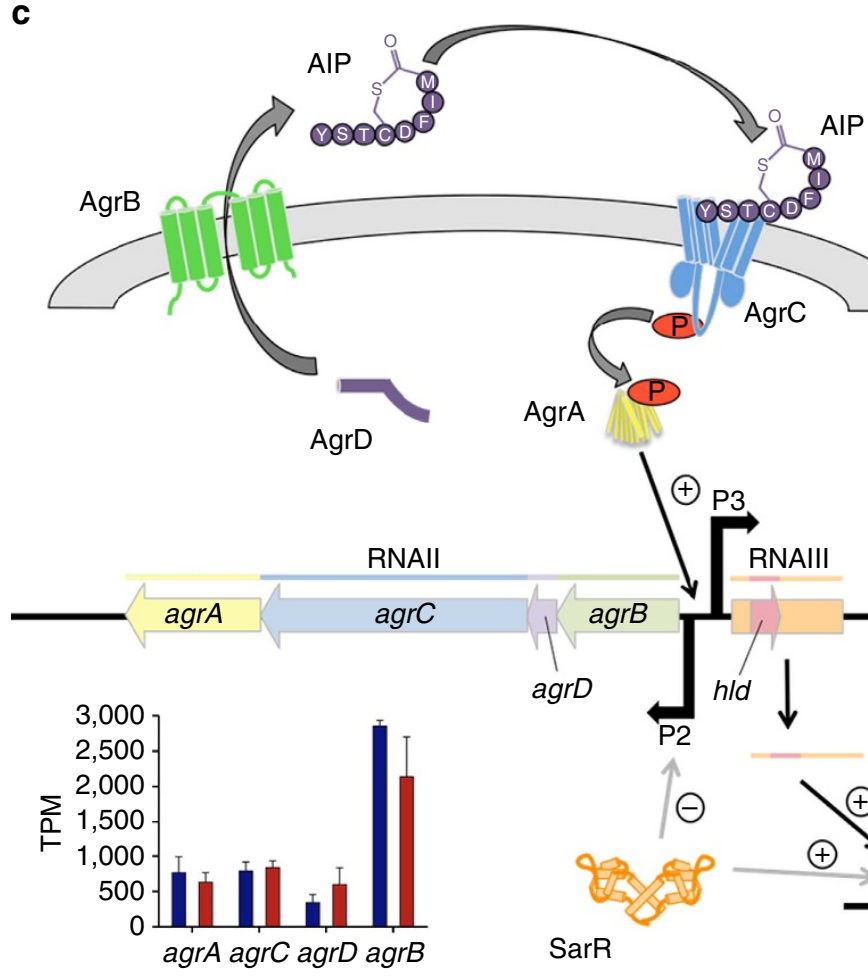

AIP

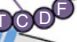

P. AgrC

AgrA

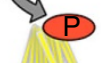

$\oplus_{\mathrm{P} 3}$

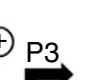

$\stackrel{\text { RNAIII }}{\longrightarrow}$

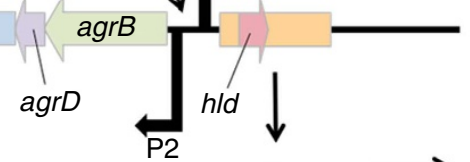

150,000

125,000

$\sum 100,000$

ㄱ 75,000 .

50,000

25,000
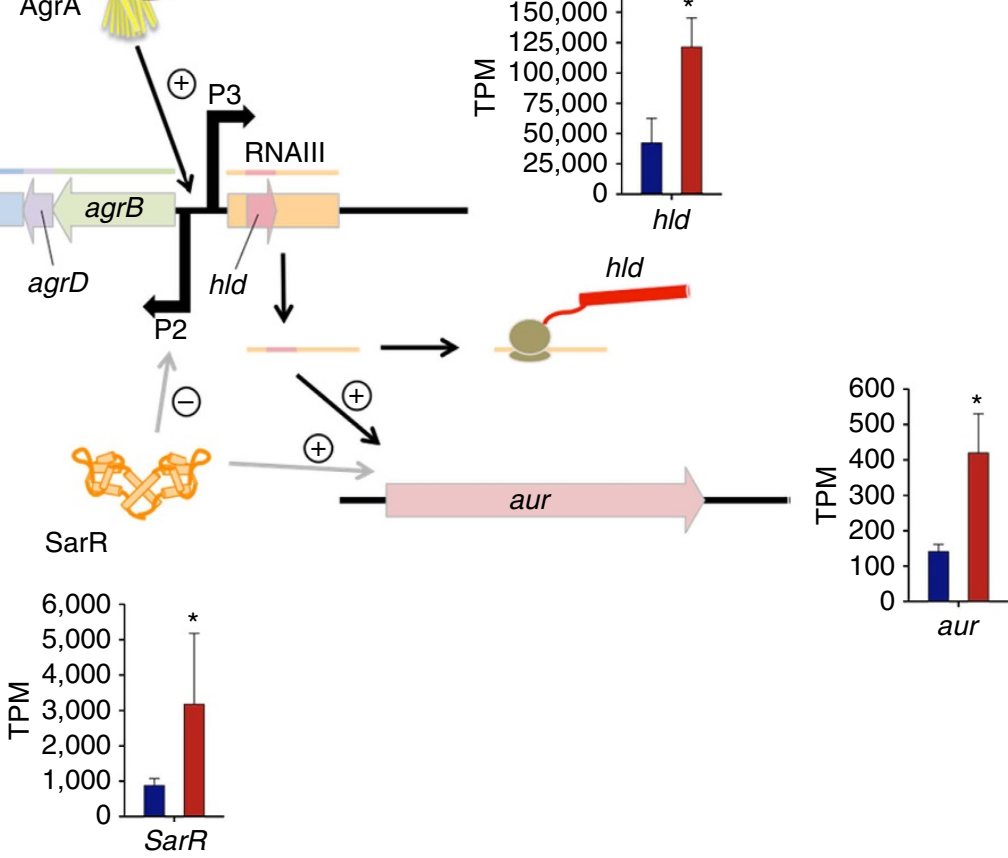

Figure 4 | Expression of selected genes by S. aureus during infection of C57BL/6 or A/J mice. (a) Expression levels of genes encoding proteases. (b) Expression levels of genes encoding toxins. (c) Schematic representation and level of expression of genes encoding the global regulator Agr and SarR. Red bars show expression values of the genes expressed by S. aureus in C57BL/6 mice and blue bars show expression values of the genes expressed by $\mathrm{S}$. aureus in $\mathrm{A} / \mathrm{J}$ mice. Each bar represents the mean TPM \pm s.d. of triplicates.

major challenge faced by $S$. aureus in susceptible A/J mice seems to be the adaptation to the adverse conditions imposed by the hyperinflammatory response and hypoxic microenvironments. To survive in the septic A/J mice, $S$. aureus increased expression of the ADI operon $(\operatorname{arc} A B D C R)$, which is generally induced under anaerobic conditions ${ }^{27}$ and is important for energy generation, but also protects $S$. aureus against acidic stress ${ }^{28}$.

The superior resistance mechanisms of C57BL/6 mice against $S$. aureus enabled a better control of bacterial multiplication.
Therefore, S. aureus faced the main challenge of counteracting the powerful host defense mechanisms of the resistant C57BL/6 mice. The transcription data indicated that $S$. aureus responded to the adverse environment encountered within C57BL/6 mice by increasing the expression of cytotoxins and extracellular proteases. Cytotoxins such as alpha-hemolysin ( $h l a)$ and PSMs (psma1-3 and hld) help S. aureus to avoid phagocytic killing by inducing pores in the membrane of host cells, leading to cell death $^{51}$ and can promote bacterial spreading by disrupting the 
a

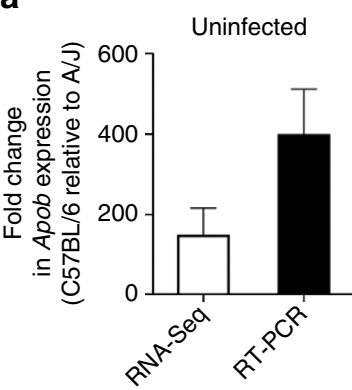

C

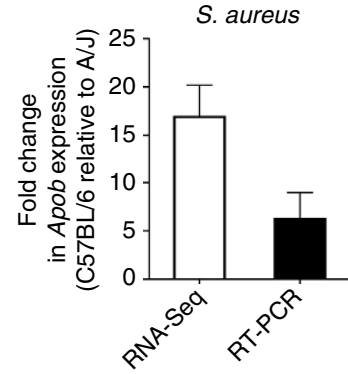

b

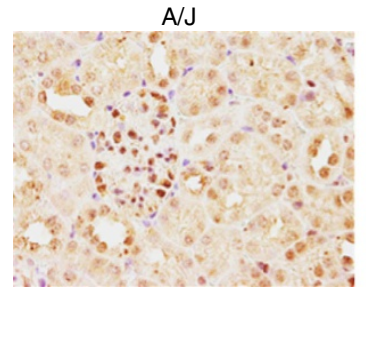

d

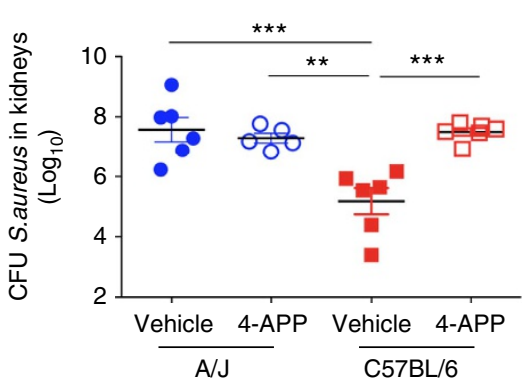

Figure 5 | Apolipoprotein B (ApoB) contributes to resistance against S. aureus bloodstream infection in C57BL/6 mice. (a) Relative fold change of Apob expression values in the kidneys of uninfected $\mathrm{C} 57 \mathrm{BL} / 6$ respect to those in the kidneys of uninfected $\mathrm{A} / \mathrm{J}$ mice determined by either RNA-Seq (white bars) or qRT-PCR (black bars). Each bar represents the mean relative fold change \pm s.d. of triplicates. (b) Expression of ApoB in kidneys of A/J (left) and C57BL/ 6 (right) mice determined by immunostaining of kidney tissue using specific antibodies against ApoB. Magnification X40. (c) Relative fold change of Apob expression values in the kidneys of S. aureus-infected C57BL/6 in comparison to those in the kidneys of S. aureus-infected A/J mice at $48 \mathrm{~h}$ of infection determined by either RNA-Seq (white bars) or qRT-PCR (black bars). Each bar represents the mean relative fold change \pm s.d. of triplicates. (d) Bacterial loads in the kidneys of A/J (blue symbols) and C57BL/6 (red symbols) mice treated with 4-Aminopyrazolo[3,4-d]pyrimidine (4-APP) (open symbols) or with vehicle alone (solid symbols) at 48 after intravenous inoculation with $2 \times 10^{7}$ CFU of S. aureus strain SH1000. Each symbol represents the bacterial counts determined in an individual mouse and the horizontal lines represent the average \pm s.d. for each mouse $\operatorname{strain}\left(n=6, t\right.$-test, $\left.{ }^{\star \star} P<0.01,{ }^{\star \star \star} P<0.001\right)$

Table 3 | S. aureus gene expression during infection of 4-APP-treated and MyD88-deficient C57BL/6 mice.

\begin{tabular}{|c|c|c|c|c|}
\hline Locus tag & $\begin{array}{l}\text { Gene } \\
\text { symbol }\end{array}$ & Description & $\begin{array}{l}\text { Relative fold change in 4-APP-treated } \\
\text { to vehicle-treated C57BL/6 mice } \\
\text { (mean (s.e.)) }\end{array}$ & $\begin{array}{l}\text { Relative fold change in MyD88- } \\
\text { deficient to wild type C57BL/6 mice } \\
\text { (mean (s.e.)) }\end{array}$ \\
\hline SAOUHSC_02265 & $\operatorname{agr} A$ & Accessory gene regulator protein $A$ & $-1.60(0.22)$ & $-1.62(0.5)$ \\
\hline SAOUHSC_02566 & sarR & Accessory regulator $\mathrm{R}$ & $-1.83(0.22)$ & $-1.23(0.09)$ \\
\hline SAOUHSC_01121 & hla & Alpha-hemolysin & $-3.32(1.56)$ & $-18.06(0.16)$ \\
\hline SAOUHSC_02971 & aur & Zinc metalloproteinase aureolysin & $-2.31(0.96)$ & $-2.59(0.75)$ \\
\hline SAOUHSC_00988 & $\operatorname{ssp} A$ & Glutamyl endopeptidase & $-2.19(0.01)$ & $-2.32(0.46)$ \\
\hline SAOUHSC_00561 & $\operatorname{vraX}$ & Protein VraX & $-3.91(2.76)$ & $1.11(0.04)$ \\
\hline SAOUHSC_00436 & gltD & Glutamate synthase subunit beta & $-2.41(1.11)$ & $-6.03(1.66)$ \\
\hline \multirow[t]{2}{*}{ SAOUHSC_01803 } & aаpA & D-serine/D-alanine/glycine transporter & $1.21(2.32)$ & $1.69(0.29)$ \\
\hline & RNAIII & Regulatory RNA & $-1.80(0.53)$ & $-2.52(0.08)$ \\
\hline
\end{tabular}

epithelial barrier ${ }^{52}$. The strong induction of genes encoding key exoproteases, including the metalloproteinase aureolysin (aur), serine proteases $(s p l A, s s p A, s p l E$ and $s p l F)$, staphostatin B ( $s p C$ ) and cysteine protease $(\operatorname{ss} p B)$, further accentuates the importance of immune evasion for $S$. aureus survival in C57BL/6 mice. These proteases can cleave and degrade components of the complement system $^{53}$ and can inhibit neutrophil chemotaxis ${ }^{54}$. The expression of these virulence determinants is orchestrated through transcriptional and post-transcriptional regulation by regulatory systems. The expression of RNAIII, a major regulator of these factors $^{38}$, was higher expressed by $S$. aureus during infection of $\mathrm{C} 57 \mathrm{BL} / 6$ mice than in $\mathrm{A} / \mathrm{J}$ mice, while the expression of the autocatalytic sensory transduction system did not differ between both mouse strains. This apparent discrepancy could, however, be explained by greater transcript abundance of SarR in S. aureus infecting C57BL/6 mice, which mitigates the expression of the agr P2 operon, while having no apparent effect on the expression of RNAIII (ref. 55). Moreover, SarR enhances the expression of genes encoding several extracellular proteases regulated by RNAIII (ref. 39) and, therefore, may act in synergy with RNAIII to boost the expression of virulence factors, required for survival under the strong immune pressure in C57BL/6 mice. The strong immune pressure in the resistant mice could also explain the greater expression of several genes of the cell wall stress stimulon by $S$. aureus infecting C57BL/6 mice. It has been shown that the magnitude of cell wall stimulon induction strongly 
a

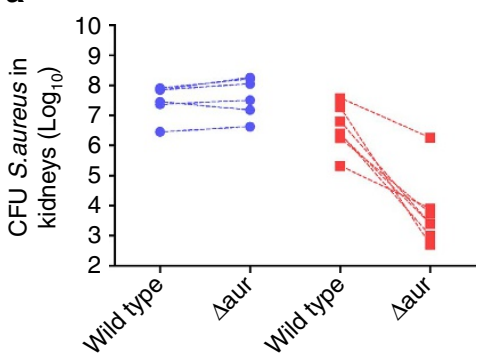

b

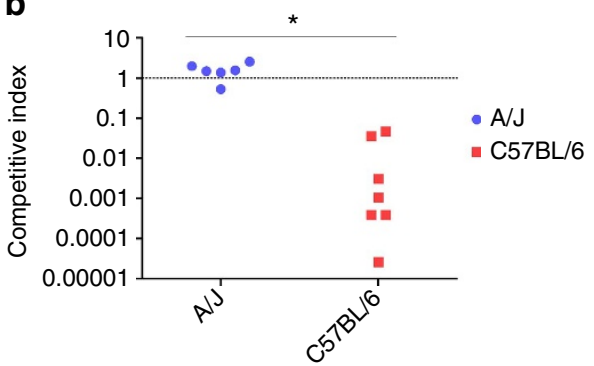

Figure 6 | In vivo competitive fitness of aureolysin-deficient (4aur) and wild type S. aureus during infection of C57BL/6 or A/J mice. (a) C57BL/6 and A/J mice were injected intravenously with a 1:1 mixtures of $\Delta$ aur and wild type S. aureus 8325-4 containing a total of approximately $4 \times 10^{7}$ bacteria. The number of bacterial cells in kidneys homogenates was determined at $48 \mathrm{~h}$ of infection. Symbols representing 4 aur and wild type $\mathrm{S}$. aureus $8325-4$ bacteria from the same animal are connected by a broken line. (b) Competitive index $(\mathrm{Cl})$, representing the ratio of the recovered $\Delta$ aur divided by the recovered wild type bacteria, within resistant $\mathrm{C} 57 \mathrm{BL} / 6$ and susceptible $\mathrm{A} / \mathrm{J}$ mice $\left(n=7, t\right.$-test, $\left.{ }^{\star} P<0.001\right)$

depends on the concentration of cell wall damaging agents ${ }^{56}$, which might indicate that $S$. aureus is exposed to a higher concentration of these damaging agents during infection of C57BL/6 than during infection of $\mathrm{A} / \mathrm{J}$ mice.

To further corroborate the strong dependence of $S$. aureus expression of virulence factors on the strength of the host immune defense we investigated the effect of reducing the innate resistance of C57BL/6 mice to $S$. aureus either by chemotherapeutic reduction of $A p o B$ concentration or by genetic deletion of the adaptor molecule MyD88 on the pathogen's gene expression. The results showed that reduction of resistance to infection in C57BL/6 mice resulted in a shift of S. aureus gene expression. The genes encoding the virulence factors alpha-hemolysin ( $h l a)$, aureolysin (aur) and a glutamyl endopeptidase (sspA) as well as the virulence regulators SarR (sarR) and RNAIII were expressed to a lesser extent by $S$. aureus infecting the more susceptible 4-APP-treated or MyD88-deficient C57BL/6 mice than during infection of normal C57BL/6 mice. Together these results provide evidence of a relationship between host resistance and pathogen expression of virulence factors.

After having demonstrated the differential expression of virulence genes by $S$. aureus in response to different levels of host resistance during infection, we investigated the impact of this dependence on the efficacy of anti-virulence approaches. Targeting aureolysin, a gene that was expressed by $S$. aureus to a higher extent during infection of $\mathrm{C} 57 \mathrm{BL} / 6$ than during infection of $\mathrm{A} / \mathrm{J}$ mice, resulted in significant reduction of $S$. aureus fitness in C57BL/6 mice $(P<0.05, t$-test), but did not affect the bacterial fitness during infection of $\mathrm{A} / \mathrm{J}$ mice. These findings highlight that the efficacy of an anti-virulence strategy against $S$. aureus will depend on the level of expression of the targeted virulence factor. Given that we show effects of differential expression of virulence factors on the efficiency of targeted interference in a defined model using two inbred mouse strains, it can be assumed that the more diverse situation in humans would even further complicate the application of anti-virulence strategies.

In summary, by combining the transcriptional response of the pathogen and of the resistant and susceptible hosts in a single experimental system, we provided evidence of the impact of host intrinsic variability on the gene expression of $S$. aureus during infection. Differences in the capacity of C57BL/6 and A/J mice to control $S$. aureus resulted in different amounts of bacteria in the organs, which might affect bacterial gene expression via growth phase as well as quorum-sensing-dependent regulation and consequently result in the different expression of virulence factors. This information is essential when searching for novel anti-virulence targets. Although a number of anti-virulence strategies targeting $S$. aureus toxins as well as the quorum-sensing regulator agr have been tested in pre-clinical mouse models with promising results ${ }^{57-59}$, a common limitation of theses studies is the determination of treatment efficacy using a single host background. The findings of our study highlight the risk of drawing definitive conclusions for in vivo efficacy from such pre-clinical studies using a single host strain and emphasize the necessity to evaluate anti-virulence strategies in a range of host backgrounds. Furthermore, heterogeneity in the expression of virulence factors within the bacterial population infecting an individual host has been demonstrated in S. aureus during in vivo infection $^{60}$. This will pose an additional problem for antivirulence strategies, since only the specific population expressing the virulence factors targeted by the anti-virulence approach will be affected, leaving intact the rest of the bacterial population. This is an important issue that needs to be addressed in future studies.

\section{Methods}

Bacterial strains. The S. aureus strains used in this study were strain SH1000 (ref. 61), the progenitor strain 8325-4 and the aureolysin-deficient (8325-4 $\Delta$ aur) S. aureus mutant strain ${ }^{62}$. Bacteria were grown to mid-log phase in brain heart infusion medium (BHI) at $37^{\circ} \mathrm{C}$ with shaking (120 r.p.m.), collected by centrifugation, washed with sterile PBS and diluted to the required concentration for injection. The number of viable bacteria was determined after serial dilution and plating on blood agar.

Mice and infection model. Pathogen-free 10 weeks old female C57BL/6 and A/J mice were purchased from Harlan-Winkelmann (Envigo, The Netherlands) and had similar body weight $(21 \pm 1.9 \mathrm{~g}$ for A/J and $20.5 \pm 0.8 \mathrm{~g}$ for C57BL/6 mice). Mice were infected intravenously with $4 \times 10^{7}$ colony forming units (CFU) of S. aureus strain SH1000 in $100 \mu \mathrm{l}$ of PBS via a lateral tail vein. For determination of bacterial numbers in the kidneys, mice were killed by $\mathrm{CO}_{2}$ asphyxiation at $48 \mathrm{~h}$ after bacterial inoculation, kidneys were removed and homogenized in PBS. Serial 10 -fold dilutions of kidney homogenate were plated on blood agar plates. Bacterial colonies were counted after incubation at $37^{\circ} \mathrm{C}$ for $24 \mathrm{~h}$ and calculated as $\mathrm{CFU}$ per kidneys.

For competition experiments, mice were intravenously inoculated with a 1:1 mixture of S. aureus strain 8325-4 and 8325-4 $\Delta$ aur mutant strain containing a total of $\sim 4 \times 10^{7}$ bacteria. Mice were killed at $48 \mathrm{~h}$ of infection and the amount of each bacterial strain was determined by plating kidney homogenates in the absence (wild type $+\Delta$ aur strain) or in the presence ( $\Delta$ aur strain) of $7.5 \mu \mathrm{g} \mathrm{ml}^{-1}$ Erythromycin. The competitive index (CI) in the mixed infection was defined as the $\Delta$ aur/wild type ratio in the infected kidneys at $48 \mathrm{~h}$ of infection. The experiment was repeated independently three times.

In some experiments, mice were treated intraperitoneally with $2.5 \mathrm{\mu g} \mathrm{ml}^{-1}$ of 4-Aminopyrazolo[3,4-d]pyrimidine (4-APP, Sigma-Aldrich) in $0.9 \% \mathrm{NaCl}$ solution or with vehicle alone $(0.9 \% \mathrm{NaCl})$ at 48 and $24 \mathrm{~h}$ before bacterial inoculation. Individual mice were randomly assigned to the treatment or control group. Bacterial injections were performed blindly. Mice were then infected with $2 \times 10^{7}$ bacteria of S. aureus strain $\mathrm{SH} 1000$, killed at $48 \mathrm{~h}$ of infection and the amount of each bacterial strain was determined by plating on blood agar. The experiment was repeated independently three times. 
Animal experiments were performed in strict accordance with the German regulations of the Society for Laboratory Animal Science (GV- SOLAS) and the European Health Law of the Federation of Laboratory Animal Science Associations (FELASA) and animals were excluded from further analysis if killing was necessary according to the human endpoints established by the ethical board. All experiments were approved by the ethical board Niedersächsisches Landesamt für Verbraucherschutz und Lebensmittelsicherheit, Oldenburg, Germany (LAVES; permit N. 33.9-42502-04-13/1195).

Sample preparation and fixation for RNA-seq. For RNA-seq experiments, three infected biological replicates from each mouse strain were analysed. Three uninfected, mock-treated replicates of each mouse strain were included as a reference to assess host gene expression changes induced by the infection. Each replicate consisted of pooled equimolar amounts of RNA extracted from the kidneys of five mice. To stabilize the RNA expression profiles, kidneys removed from infected and uninfected $\mathrm{A} / \mathrm{J}$ and $\mathrm{C} 57 \mathrm{BL} / 6$ mice were stored in $3 \mathrm{ml}$ of RNAlater (Ambion) at $4{ }^{\circ} \mathrm{C}$ overnight.

Total RNA extraction, purification and rRNA depletion. Whole kidneys were mechanically disrupted using a Polytron disperser (Kinematica) in $2 \mathrm{ml}$ of sterile PBS supplemented with $1 \% \beta$-mercaptoethanol (Sigma-Aldrich) to denature RNases released on cell disruption and processed as previously described ${ }^{63}$. Samples were further treated using lysing matrices B (MP Biomedicals) to achieve efficient lysis of bacterial cells released from the homogenized tissue Total RNA was purified from the disrupted samples using a classical phenol: chloroform:isoamyl alcohol method and concentrated by ethanol precipitation. Contaminating genomic DNA was removed by DNase treatment using $4 \mathrm{U}$ Turbo DNase (Ambion) on $5 \mu \mathrm{g}$ of total RNA for $30 \mathrm{~min}$ at $37^{\circ} \mathrm{C}$. To limit RNA degradation, $40 \mathrm{U}$ of RiboGuard RNase Inhibitor (Epicentre) were added to the samples. RNA integrity was checked using an Agilent 2100 Bioanalyzer (Agilent Technologies). ERCC RNA Spike-In Mixes (Thermo Fisher Scientific) were added according to the manufacturer's guidelines to determine the dynamic range and lower limit of detection of the Illumina RNA-sequencing platform and control for experimentally derived errors in the generated datasets. DNA-depleted RNA samples were then depleted of murine and $S$. aureus ribosomal RNA using the RiboZero epidemiology kit (Epicenter) according to the manufacturer's protocol. Following ethanol precipitation at $-20^{\circ} \mathrm{C}$ for $5 \mathrm{~h}$, RNA quantity and rRNA depletion efficiency were accessed using the Agilent 2100 Bioanalyzer (Agilent Technologies) and NanoDrop 1000 spectrophotometer (Thermo Scientific).

Illumina library preparation and sequencing. The cDNA libraries were prepared using the ScriptSeq v2 RNA-seq library preparation kit (Epicentre) according to the manufacturer's instructions. Briefly, $50 \mathrm{ng}$ of RNA were fragmented in fragmentation solution for $5 \mathrm{~min}$ at $85^{\circ} \mathrm{C}$ and cDNA was synthesized using random hexamer primer including a $5^{\prime}$-end tagging sequence. RNA was removed and the 3 '-tagged cDNA was synthesized via Terminal-Tagging Oligos. The generated di-tagged cDNA was purified using the MinElute PCR Purification kit according to the manufacturer's instructions. Subsequently, adaptor-tagged RNA-Seq libraries were generated by amplification of the di-tagged cDNA using primers that add indices for sample multiplexing and Illumina adaptor sequences. Following removal of excess PCR Primers via Exonuclease I treatment, the generated libraries were purified using the MinElute PCR Purification kit according to the manufacturer's instructions. The cDNA libraries were size-selected by agarose gel electrophoresis and fragments between 180 and $650 \mathrm{bp}$ were enriched by gel extraction using the QIAquick gel extraction kit to exclude remaining contamination by other RNA species. Library quality and size-selection was assessed using the Agilent 2100 Bioanalyzer. The cDNA libraries were sequenced using single-end sequencing (50 bp) on the Illumina HiSeq 2500 platform utilizing the TruSeq S.R. cluster kit, v3-cBot-HS (Illumina). Two libraries were multiplexed per lane and sequenced to 58 cycles in one direction. Between 84 and 139 million reads were recorded per library.

Data processing. Raw sequenced reads were quality filtered and trimmed for Illumina-adapter contamination using fastq-mcf ${ }^{64}$. Remaining reads were aligned either to the genome of the $S$. aureus strain 8325-4, manually revised for the changes of strain SH1000 (ref. 21), or to the Mus musculus reference genome GRcm38.p3 using STAR ${ }^{65}$. Minimal sequence matches of 30 nucleotides with a minimal sequence homology of $93,33 \%$ were included. Mapped reads were collapsed using samtools ${ }^{66}$ and counted using HTSeq ${ }^{67}$. Raw mapped read counts were normalized to transcripts per million (TPM).

Quantitative RT-PCR. Total RNA was extracted from tissue samples using the phenol:chloroform:isoamyl alcohol method. DNA was removed by DNase treatment utilizing the Turbo DNA-free kit (Invitrogen). RNA samples were reverse transcribed and amplified using a SensiFAST SYBR No-ROX Kit (Bioline) following the manufacturer's recommendations. The primers used for quantitative RT-PCR are provided in Supplementary Table 3. Thermal cycling conditions for hld/RNAIII, sarR, hla, sspA, aur, vraX, gltB, aapA, apoB and $\beta$-actin quantification consisted of reverse transcription for $20 \mathrm{~min}$ at $45^{\circ} \mathrm{C}$, initial denaturation for $5 \mathrm{~min}$ at $95^{\circ} \mathrm{C}$, followed by 40 cycles of $20 \mathrm{~s}$ at $95^{\circ} \mathrm{C}$ (denaturation), $20 \mathrm{~s}$ at $60^{\circ} \mathrm{C}$ (annealing) and $20 \mathrm{~s}$ at $72^{\circ} \mathrm{C}$ (elongation). Annealing temperature for the $16 \mathrm{~s}$ primers was $70^{\circ} \mathrm{C}$. Data were normalized against the housekeeping gene $16 \mathrm{~s}$ for S. aureus and $\beta$-actin for $a p o B$. Fold change values were calculated by the Pfaffl equation, in which the expression ratio is estimated by $\left(\mathrm{E}_{\text {target }}\right){ }^{\prime C \mathrm{Ct}}$ target

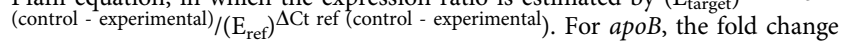
between the expression of apoB in the kidneys of C57BL/6 was expressed relative to that in $\mathrm{A} / \mathrm{J}$ mice. For $S$. aureus values were expressed as the fold change between the expression of a specific gene by $S$. aureus during the infection of 4-APP-treated C57BL/6 mice in respect to the expression by S. aureus during infection of vehicletreated $\mathrm{C} 57 \mathrm{BL} / 6$ mice.

Apolipoprotein B immunohistochemistry. Kidney samples were collected from uninfected $\mathrm{C} 57 \mathrm{BL} / 6$ and $\mathrm{A} / \mathrm{J}$ mice for immunohistochemistry. Tissues were fixed in $10 \%$ formalin and embedded in paraffin. Immunohistochemistry was performed using a polyclonal rabbit anti-mouse apolipoprotein B-specific antibody (Abcam ab20737). Paraffin sections were rehydrated through graded alcohols. For blocking of the endogenous peroxidase, formalin-fixed, paraffin-embedded tissue sections were treated with $0.5 \% \mathrm{H}_{2} \mathrm{O}_{2}$ diluted in methanol for $30 \mathrm{~min}$ at room temperature (RT). Subsequently, sections were heated in $10 \mathrm{mM} \mathrm{Na}$-citrate buffer $\mathrm{pH} 6.0$ for $20 \mathrm{~min}$ in a microwave oven $(800 \mathrm{~W})$. Following incubation with $20 \%$ goat serum (obtained from University of Veterinary Medicine Hannover) for $30 \mathrm{~min}$ to block non-specific binding sites, sections were incubated with polyclonal rabbit antimouse apolipoprotein B-specific antibody (Abcam ab20737, dilution 1:50) for $1.5 \mathrm{~h}$ at RT. Rabbit serum (Sigma-Aldrich; R 4505) was used as negative control. Thereafter, sections were treated for $30 \mathrm{~min}$ at RT with the secondary goat anti-rabbit antibody (Vector Laboratories BA1000, dilution 1:200). Slides were subsequently incubated with the peroxidase-conjugated avidin-biotin complex (Vector Laboratories PK6100) for $30 \mathrm{~min}$ at RT. All antibodies were diluted in PBS After visualization of the positive antigen-antibody reaction by incubation with 3.3-diaminobenzidine-tetrachloride (Fluka 32750) for $5 \mathrm{~min}$, sections were counterstained with hematoxylin.

Tissue ELISA. Kidneys were taken from S. aureus-infected C57BL/6 and A/J mice at $48 \mathrm{~h}$ after bacterial inoculation or from mock-treated and homogenized in tissue lysis buffer containing $20 \mathrm{mM} \mathrm{NaCl}, 5 \mathrm{mM}$ EDTA, $10 \mathrm{mM}$ Tris, $1 \mathrm{mM}$ PMSF, $1 \mu \mathrm{g} / \mathrm{ml}$ Leupeptin, $28 \mu \mathrm{g} / \mathrm{ml}$ Aprotinin at $\mathrm{pH}$ 7.4. Homogenized samples were centrifuged twice and the supernatant was collected and used for determination of interleukin 6 (IL-6), interleukin $1 \beta$ (IL-1 $\beta$ ), interleukin 10 (IL-10), Cxcl1, Cxcl2 and $\mathrm{Ccl} 2$ concentrations using matched antibody pairs and recombinant proteins as standards. Briefly, 96-well microtiter plates were coated overnight at $4{ }^{\circ} \mathrm{C}$, with the corresponding purified anti-mouse capture monoclonal antibody (Biolegend 431305, 432602, 431412, R\&D Systems DY452, DY453, BD Bioscience 555260) in the coating buffer $\left(\mathrm{Na}_{3} \mathrm{PO}_{4} \mathrm{pH} 6.5, \mathrm{Na}_{2} \mathrm{CO}_{3} \mathrm{pH} 9.5\right.$ or PBS). Plates were washed and blocked with $1 \%$ bovine serum albumin-PBS or $10 \%$ fetal bovine serum-PBS. Tissue supernatants as well as standard were added in technical triplicates at previously established dilutions. Monoclonal anti-mouse detection antibody was added incubated for $1 \mathrm{~h}$ at $37^{\circ} \mathrm{C}$, washed and further incubated with avidinhorseradish peroxidase for detection (this step was omitted for Ccl2 quantification since the detection antibody was already HRP coupled). Plates were developed using TMB as substrate.

Statistical analysis. Statistical analyses were performed using PRIMER (Version 6.1.6, PRIMER-E; Plymouth Marine Laboratory), GraphPad Prism (Version 5.04, GraphPad Software, Inc.) and R (http://www.r-project.org). To assess the global similarity between biological replicates, a sample-similarity matrix was generated using euclidean distance measurement. The transcriptional profiles were compared using PCA and group-average agglomerative hierarchical clustering. PERMANOVA was used to determine the statistical significance of differences between the transcriptional profiles of the different groups. For the one-way PERMANOVA, the resemblance matrix was generated using euclidean distance measurement using type III (partial) sums of squares with a fixed effects sum to zero for mixed terms. Exact $P$ values were generated using unrestricted permutation of the raw data. Pseudo-F statistic and generated $P$ values were reported for each pair of conditions. Monte Carlo simulations were performed in the pairwise test function if low permutations were obtained. Differences between groups were considered significant if $P<0.05$. Low read counts were filtered from the murine datasets applying a strict LODR-cutoff (limit of detection of ratio) as determined for a two-fold increase in transcript abundance by analysis of the ERCC spike-in mixes. To avoid misinterpretation resulting from the low sequencing depth of the S. aureus transcriptomes, a more stringent threshold was applied, where genes with transcripts levels below $0.05 \%$ ( $<16$ copies per 33,228 reads) were filtered from the bacterial gene expression datasets. Due to the differences in sequencing depth different strategies were used for determination of differential gene expression analysis for host and pathogen. To identify genes differentially expressed by S. aureus between infection of $\mathrm{A} / \mathrm{J}$ and $\mathrm{C} 57 \mathrm{BL} / 6$ mice, the non-parametric NOISeq algorithm, whose sensitivity is less depended on the sequencing depth compared to other methods ${ }^{68}$, was applied. A gene was considered differentially expressed by 
S. aureus between infection of $\mathrm{A} / \mathrm{J}$ and $\mathrm{C} 57 \mathrm{BL} / 6$ mice when the FDR was $<0.05$ (probability value $>0.95$ ). The DESeq2 algorithm ${ }^{22}$ was applied to identify genes differentially expressed between uninfected and S. aureus-infected $\mathrm{A} / \mathrm{J}$ and C57BL/6 mice. A gene was considered significantly differential expressed between mice if the Benjamini-Hochberg adjusted $P$ value was $<0.05$ and the $\log _{2}$ fold change $\geq 1$. KEGG pathway enrichment analysis was performed using DAVID Informatics Resources ${ }^{69}$. KEGG pathways with a FDR $\leq 0.05$ were considered significantly enriched.

Data availability. The RNA-seq data that support the findings of this study have been deposited in the European Nucleotide Archive with the accession code PRJEB14649. All other relevant data are available from the corresponding author on request.

\section{References}

1. Spellberg, B. et al. The epidemic of antibiotic-resistant infections: a call to action for the medical community from the Infectious Diseases Society of America. Clin. Infect. Dis. 46, 155-164 (2008).

2. Rasko, D. A. \& Sperandio, V. Anti-virulence strategies to combat bacteriamediated disease. Nat. Rev. Drug Discov. 9, 117-128 (2010).

3. Wang, J. et al. Novel inhibitor discovery and the conformational analysis of inhibitors of listeriolysin O via protein-ligand modeling. Sci. Rep. 5, 8864 (2015).

4. Krachler, A. M. \& Orth, K. Targeting the bacteria-host interface: strategies in anti-adhesion therapy. Virulence 4, 284-294 (2013).

5. Baron, C. Antivirulence drugs to target bacterial secretion systems. Curr. Opin. Microbiol. 13, 100-105 (2010).

6. Rasko, D. A. et al. Targeting QseC signaling and virulence for antibiotic development. Science 321, 1078-1080 (2008).

7. Starkey, M. et al. Identification of anti-virulence compounds that disrupt quorum-sensing regulated acute and persistent pathogenicity. PLoS Pathog. 10, e1004321 (2014).

8. Casadevall, A. \& Pirofski, L. Host-pathogen interactions: the attributes of virulence. J. Infect. Dis. 184, 337-344 (2001).

9. Lowy, F. D. Staphylococcus aureus infections. N. Engl. J. Med. 339, 520-532 (1998).

10. Hiramatsu, K. et al. Multi-drug-resistant Staphylococcus aureus and future chemotherapy. J. Infect. Chemother. 20, 593-601 (2014).

11. Fowler, Jr V. G. \& Proctor, R. A. Where does a Staphylococcus aureus vaccine stand? Clin. Microbiol. Infect. 20(Suppl 5): 66-75 (2014).

12. Kong, C., Neoh, H. M. \& Nathan, S. Targeting Staphylococcus aureus toxins: a potential form of anti-virulence therapy. Toxins (Basel) 8, pii E72 (2016).

13. Fitzgerald-Hughes, D., Devocelle, M. \& Humphreys, H. Beyond conventional antibiotics for the future treatment of methicillin-resistant Staphylococcus aureus infections: two novel alternatives. FEMS Immunol. Med. Microbiol. 65, 399-412 (2012).

14. Mavromatis, C. H. et al. The co-transcriptome of uropathogenic Escherichia coli-infected mouse macrophages reveals new insights into host-pathogen interactions. Cell Microbiol. 17, 730-746 (2015).

15. Humphrys, M. S. et al. Simultaneous transcriptional profiling of bacteria and their host cells. PLoS ONE 8, e80597 (2013).

16. von Kockritz-Blickwede, M. et al. Immunological mechanisms underlying the genetic predisposition to severe Staphylococcus aureus infection in the mouse model. Am. J. Pathol. 173, 1657-1668 (2008).

17. Ahn, S. H. et al. Two genes on A/J chromosome 18 are associated with susceptibility to Staphylococcus aureus infection by combined microarray and QTL analyses. PLoS Pathog. 6, e1001088 (2010).

18. Westermann, A. J., Gorski, S. A. \& Vogel, J. Dual RNA-seq of pathogen and host. Nat. Rev. Microbiol. 10, 618-630 (2012).

19. Westermann, A. J. et al. Dual RNA-seq unveils noncoding RNA functions in host-pathogen interactions. Nature 529, 496-501 (2016).

20. Baddal, B. et al. Dual RNA-seq of nontypeable Haemophilus influenzae and host cell transcriptomes reveals novel insights into host-pathogen cross talk. MBio. 6, e01765-15 (2015).

21. O'Neill, A. J. Staphylococcus aureus SH1000 and 8325-4: comparative genome sequences of key laboratory strains in staphylococcal research. Lett. Appl. Microbiol. 51, 358-361 (2010).

22. Love, M. I., Huber, W. \& Anders, S. Moderated estimation of fold change and dispersion for RNA-seq data with DESeq2. Genome Biol. 15, 550 (2014).

23. Okamoto, K., Tamura, T. \& Sawatsubashi, Y. Sepsis and disseminated intravascular coagulation. J. Intensive Care 4, 23 (2016).

24. Nizet, V. \& Johnson, R. S. Interdependence of hypoxic and innate immune responses. Nat. Rev. Immunol. 9, 609-617 (2009).

25. Imtiyaz, H. Z. \& Simon, M. C. Hypoxia-inducible factors as essential regulators of inflammation. Curr. Top. Microbiol. Immunol. 345, 105-120 (2010).

26. Yang, Z. \& Ming, X. F. Arginase: the emerging therapeutic target for vascular oxidative stress and inflammation. Front. Immunol. 4, 149 (2013).
27. Makhlin, J. et al. Staphylococcus aureus ArcR controls expression of the arginine deiminase operon. J. Bacteriol. 189, 5976-5986 (2007).

28. Hall, J. W. \& Ji, Y. Sensing and Adapting to Anaerobic Conditions by Staphylococcus aureus. Adv. Appl. Microbiol. 84, 1-25 (2013).

29. Seidl, K. et al. Effect of a glucose impulse on the CcpA regulon in Staphylococcus aureus. BMC Microbiol. 9, 1 (2009).

30. Monnet, V. Bacterial oligopeptide-binding proteins. Cell Mol. Life Sci. 60, 2100-2114 (2003).

31. Kolar, S. L. et al. Extracellular proteases are key mediators of Staphylococcus aureus virulence via the global modulation of virulence-determinant stability. Microbiologyopen 2, 18-34 (2013).

32. Jusko, M. et al. Staphylococcal proteases aid in evasion of the human complement system. J. Innate Immun. 6, 31-46 (2013).

33. Ko, Y. P. et al. Phagocytosis escape by a Staphylococcus aureus protein that connects complement and coagulation proteins at the bacterial surface. PLoS Pathog. 9, e1003816 (2013).

34. Vandenesch, F., Lina, G. \& Henry, T. Staphylococcus aureus hemolysins, bi-component leukocidins, and cytolytic peptides: a redundant arsenal of membrane-damaging virulence factors? Front. Cell. Infect. Microbiol. 2, 12 (2012).

35. Bronner, S., Monteil, H. \& Prevost, G. Regulation of virulence determinants in Staphylococcus aureus: complexity and applications. FEMS Microbiol. Rev. 28, 183-200 (2004).

36. Peng, H. L., Novick, R. P., Kreiswirth, B., Kornblum, J. \& Schlievert, P. Cloning, characterization, and sequencing of an accessory gene regulator (agr) in Staphylococcus aureus. J. Bacteriol. 170, 4365-4372 (1988).

37. Janzon, L., Lofdahl, S. \& Arvidson, S. Identification and nucleotide sequence of the delta-lysin gene, hld, adjacent to the accessory gene regulator (agr) of Staphylococcus aureus. Mol. Gen. Genet. 219, 480-485 (1989).

38. Novick, R. P. et al. Synthesis of staphylococcal virulence factors is controlled by a regulatory RNA molecule. EMBO J. 12, 3967-3975 (1993).

39. Gustafsson, E. \& Oscarsson, J. Maximal transcription of aur (aureolysin) and sspA (serine protease) in Staphylococcus aureus requires staphylococcal accessory regulator R (sarR) activity. FEMS Microbiol. Lett. 284, 158-164 (2008).

40. Midorikawa, K. et al. Staphylococcus aureus susceptibility to innate antimicrobial peptides, beta-defensins and CAP18, expressed by human keratinocytes. Infect. Immun. 71, 3730-3739 (2003).

41. Ernst, C. M. \& Peschel, A. Broad-spectrum antimicrobial peptide resistance by MprF-mediated aminoacylation and flipping of phospholipids. Mol. Microbiol. 80, 290-299 (2011).

42. Peterson, M. M. et al. Apolipoprotein B is an innate barrier against invasive Staphylococcus aureus infection. Cell Host Microbe 4, 555-566 (2008).

43. Manifold-Wheeler, B. C. et al. Serum lipoproteins are critical for pulmonary innate defense against Staphylococcus aureus quorum sensing. J. Immunol. 196, 328-335 (2016).

44. Mounkes, L. C. et al. Evaluation of the role of lipoprotein metabolism genes in systemic cationic liposome-mediated gene transfer in vivo. Hum. Gene Ther. 12, 1939-1954 (2001).

45. Takeuchi, O., Hoshino, K. \& Akira, S. Cutting edge: TLR2-deficient and MyD88-deficient mice are highly susceptible to Staphylococcus aureus infection. J. Immunol. 165, 5392-5396 (2000).

46. Laarman, A. J. et al. Staphylococcus aureus metalloprotease aureolysin cleaves complement C3 to mediate immune evasion. J. Immunol. 186, 6445-6453 (2011).

47. Cassat, J. E. et al. A secreted bacterial protease tailors the Staphylococcus aureus virulence repertoire to modulate bone remodeling during osteomyelitis. Cell Host Microbe 13, 759-772 (2013).

48. Cegelski, L., Marshall, G. R., Eldridge, G. R. \& Hultgren, S. J. The biology and future prospects of antivirulence therapies. Nat. Rev. Microbiol. 6, 17-27 (2008).

49. Weidemann, A. \& Johnson, R. S. Biology of HIF-1alpha. Cell Death Differ. 15, 621-627 (2008).

50. Zinkernagel, A. S., Johnson, R. S. \& Nizet, V. Hypoxia inducible factor (HIF) function in innate immunity and infection. J. Mol. Med. (Berl) 85, 1339-1346 (2007).

51. Otto, M. Staphylococcus aureus toxins. Curr Opin Microbiol. 17, 32-37 (2014).

52. Inoshima, I. et al. A Staphylococcus aureus pore-forming toxin subverts the activity of ADAM10 to cause lethal infection in mice. Nat. Med. 17, 1310-1314 (2011).

53. Jusko, M. et al. Staphylococcal proteases aid in evasion of the human complement system. J. Innate Immun. 6, 31-46 (2014).

54. Smagur, J. et al. Staphylococcal cysteine protease staphopain B ( $\mathrm{SspB}$ ) induces rapid engulfment of human neutrophils and monocytes by macrophages. Biol. Chem. 390, 361-371 (2009).

55. Reyes, D. et al. Coordinated regulation by AgrA, SarA, and SarR to control agr expression in Staphylococcus aureus. J. Bacteriol. 193, 6020-6031 (2011). 
56. Dengler, V., Meier, P. S., Heusser, R., Berger-Bächi, B. \& McCallum, N. Induction kinetics of the Staphylococcus aureus cell wall stress stimulon in response to different cell wall active antibiotics. BMC Microbiol. 11, 1 (2011).

57. Hua, L. et al. Assessment of an anti-alpha-toxin monoclonal antibody for prevention and treatment of Staphylococcus aureus-induced pneumonia. Antimicrob. Agents Chemother. 58, 1108-1117 (2014).

58. Tkaczyk, C. et al. Identification of anti-alpha toxin monoclonal antibodies that reduce the severity of Staphylococcus aureus dermonecrosis and exhibit a correlation between affinity and potency. Clin. Vaccine Immunol. 19, 377-385 (2012).

59. Simonetti, O. et al. RNAIII-inhibiting peptide enhances healing of wounds infected with methicillin-resistant Staphylococcus aureus. Antimicrob. Agents Chemother. 52, 2205-2211 (2008).

60. Liese, J., Rooijakkers, S. H., Strijp, J. A., Novick, R. P. \& Dustin, M. L. Intravital two-photon microscopy of host-pathogen interactions in a mouse model of Staphylococcus aureus skin abscess formation. Cell Microbiol. 15, 891-909 (2013).

61. Horsburgh, M. J. et al. $\sigma \mathrm{B}$ modulates virulence determinant expression and stress resistance: characterization of a functional rsbU strain derived from Staphylococcus aureus 8325-4. J. Bacteriol. 184, 5457-5467 (2002).

62. Shaw, L., Golonka, E., Potempa, J. \& Foster, S. J. The role and regulation of the extracellular proteases of Staphylococcus aureus. Microbiology 150, 217-228 (2004).

63. Szafranska, A. K. et al. High-resolution transcriptomic analysis of the adaptive response of Staphylococcus aureus during acute and chronic phases of osteomyelitis. MBio. 5, e01775-14 (2014).

64. Aronesty, E. Comparison of sequencing utility programs. Open Bioinforma. J. 7, no. 1 (2013)

65. Dobin, A. et al. STAR: ultrafast universal RNA-seq aligner. Bioinformatics 29, 15-21 (2013).

66. Li, H. et al. The sequence alignment/map format and SAMtools. Bioinformatics 25, 2078-2079 (2009).

67. Anders, S., Pyl, P. T. \& Huber, W. HTSeq-a Python framework to work with high-throughput sequencing data. Bioinformatics 31, 166-169 (2015).

68. Tarazona, S., García, F., Ferrer, A., Dopazo, J. \& Conesa, A. NOIseq: a RNA-seq differential expression method robust for sequencing depth biases. EMBnet. journal 17, 18-19 (2012).

69. Huang, D. W., Sherman, B. T. \& Lempicki, R. A. Bioinformatics enrichment tools: paths toward the comprehensive functional analysis of large gene lists. Nucleic Acids Res. 37, 1-13 (2009).

\section{Acknowledgements}

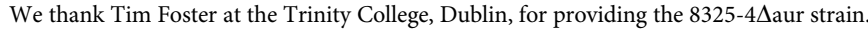
We thank Dr Anne Fiebig at the Helmholtz Center for Infection Research, Braunschweig for help with the bioinformatic analysis and Maria Voigt at the German Centre for Integrative Biodiversity Research (iDiv), Leipzig, for critical review of the manuscript. R.T. was partially supported by the President's Initiative and Network Fund of the Helmholtz Association of German Research Centres (HGF) under contract number VH-GS-202.

\section{Author contributions}

R.T. and E.M. designed the study. R.T., O.G. and A.B. performed the experiments and analysis of the data. R.T. and E.M. wrote the manuscript.

\section{Additional information}

Supplementary Information accompanies this paper at http://www.nature.com/ naturecommunications

Competing financial interests: The authors declare no competing financial interests.

Reprints and permission information is available online at http://npg.nature.com/ reprintsandpermissions/

How to cite this article: Thänert, R. et al. Host-inherent variability influences the transcriptional response of Staphylococcus aureus during in vivo infection. Nat. Commun 8, 14268 doi: $10.1038 /$ ncomms14268 (2017)

Publisher's note: Springer Nature remains neutral with regard to jurisdictional claims in published maps and institutional affiliations.

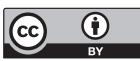

This work is licensed under a Creative Commons Attribution 4.0 International License. The images or other third party material in this article are included in the article's Creative Commons license, unless indicated otherwise in the credit line; if the material is not included under the Creative Commons license, users will need to obtain permission from the license holder to reproduce the material. To view a copy of this license, visit http://creativecommons.org/licenses/by/4.0/

C) The Author(s) 2017 